\title{
Our initial experience with breast tissue marker
}

\begin{abstract}
Breast tissue markers are novel in the field of breast intervention. They help to well localize the breast lesions that have been biopsied or have been subjected to neoadjuvant chemotherapy as these lesions might become quite inconspicuous on imaging. They are not only a guide for radiologists but also for surgeons to extract the entire lesion, for oncologists to evaluate treatment response and for pathologists to assess the adequacy of the tissue they sampled. They are important tools for follow up of breast lesions. Newer markers are available which are not just visible on mammogram but also on ultrasonography.
\end{abstract}

We had deployed four UltraClip ${ }^{\circledR}$ dual trigger breast tissue markers around a malignant breast mass prior to neoadjuvant chemotherapy. Post procedure, these markers were visible as hyperechoic linear structures on ultrasonography and as ribbon shaped clips around the lesion on mammogram. They are expected to be a guide for future localization of the breast mass and assess its treatment response. Breast tissue markers are thus definitely a step towards progress in diagnosis and treatment of breast carcinoma.

Keywords: breast tissue markers, novel, ultraclip ${ }^{\circledR}$, breast carcinoma
Volume 5 Issue 6 - 2018

\author{
Smily Sharma,' Pankaj Sharma,' Udit \\ Chauhan,' Rajesh Kumar Pasricha ${ }^{2}$ \\ 'Department of Radiodiagnosis and Imaging, AllMS Rishikesh, \\ India \\ ${ }^{2}$ Department of Radiotherapy,AlIMS Rishikesh, India
}

Correspondence: Pankaj Sharma, Department of Radiodiagnosis and Imaging, AlIMS Rishikesh, B 78, Sector 23, NOIDA-20I30I, India, Tel (09I) 099I066|497, 0120-4I09552, Email pamkajrad7477@yahoo.com

Received: July 31, 2018 | Published: November 26, 2018

\section{Introduction}

With increasing burden of breast carcinoma, research continues to grow to find newer techniques for better visualization, diagnosis and treatment of breast lesions. Biopsy is the gold standard for diagnosis of breast carcinoma. However after breast biopsy or even natively, the lesion may become almost inconspicuous hindering its visualization on post procedural imaging. Also with neoadjuvant chemotherapy, the lesion shrinks and may become too vague to be defined on presurgical imaging. ${ }^{1}$

Breast tissue markers seem to be a good solution for this problem. This approach helps in relocalization of breast lesions if we want to do repeat biopsy. They are also extremely useful for preoperative hook wire placement so that the surgeon can accurately identify the lesion. This has been traditionally done on stereotaxis. However with availability of markers which can be visible on ultrasonography as well, the risk of radiation exposure further gets reduced. Moreover, ultrasonography is easily available and comfortable for the patient. These markers when used along with intraoperative ultrasound may replace other pre-operative localisation procedures in totality. ${ }^{2}$ One such marker is UltraClip ${ }^{\circledR}$ dual trigger breast tissue marker. It is an ultrasound enhanced ribbon shaped marker. It can be visible on mammography as well as ultrasonography. It also helps in correlating visualization of breast lesions on different imaging modalities. ${ }^{3}$

\section{Case report}

A 38 year old female had complaint of lump in right breast for about 2 months. There was no history of pain, nipple discharge or skin/nipple retraction. She had family history of carcinoma breast (in paternal aunt) and carcinoma uterus (in mother). On examination, there was a $3 \times 4 \mathrm{~cm}$ hard breast lump in upper outer quadrant of right breast which was non tender and was non adherent to skin or chest wall. Bilateral nipple areola complex were normal. No axillary lymphadenopathy was noticed.
She underwent digital mammography and ultrasonography examinations of bilateral breasts and axilla. Bilateral breast parenchyma were heterogeneously dense, which might obscure masses (according to ACR BIRADS 2013 classification of breast composition). ${ }^{4}$ There was an irregular high density mass with obscured margins in upper outer quadrant of right breast with surrounding architectural distortion. No calcifications were seen in the mass. There was no skin thickening or nipple retraction. No axillary lymphadenopathy was seen.

On ultrasonography examination, there was a circumscribed, irregular, hypoechoic mass with lobulated margins at 100' clock position in right breast approximately $6 \mathrm{~cm}$ away from nipple and $2 \mathrm{~cm}$ deep to skin. The mass measured approximately $4 \times 3.2 \times 2.4 \mathrm{~cm}$ and showed mild internal vascularity. The lesion was parallel in orientation and showed no posterior acoustic enhancement or shadowing. There were no echogenic foci in the mass or surrounding it. Nipple areola complex were normal. Left breast was normal. Bilateral axilla were clear of lymph nodes. Right breast was labelled as BIRADS 4B with moderate $(>10 \%$ to $<$ and equal to $50 \%)$ suspicion for malignancy. She was advised for histopathological correlation (Figure 1-4).

Trucut biopsy from right breast mass revealed invasive ductal carcinoma.Thereafter, she underwent ultrasonography examination of whole abdomen where an intramural fibroid of size approximately $4.2 \times 4.6 \mathrm{~cm}$ was seen in posterior myometrium. No skeletal metastasis were seen on bone scan. Biochemical parameters were within normal limits. She was planned for four cycles of neoadjuvant chemotherapy (NACT) followed by response assessment. Prior to the first cycle, it was decided to deploy breast tissue markers around the mass so that adequate response assessment of the tumour was possible after NACT.

Informed written consent was obtained and necessary preprocedural investigations were done. Under all aseptic precautions and local anaesthesia, ultrasound guidance was used to deploy Ultraclips at all the four corners of the right breast mass. High frequency ultrasound probe (frequency approximately 9 to $12 \mathrm{Mega} \mathrm{Hz}$ ) of GE LOGIQ S8 Ultrasound machine was used to localize the lesion and guide 
the procedure. Using four separate disposable $17 \mathrm{Gx} 12 \mathrm{~cm}$ BARD Ultraclip Dual Triggers, the lesion was pierced one after the other at all the corners. One handed single step process was used to deploy $3 \mathrm{~mm}$ sized titanium breast tissue markers. The markers were MRI compatible and were interwoven with non-absorbable polyvinyl alcohol polymer for permanent ultrasound enhancement. These could show up on ultrasonography, mammography and computed tomography. The markers were designed in such a way that they will attach to breast parenchyma and hence will not migrate.
Post procedural confirmation of the ultraclips was done on ultrasonography where approximately $3 \mathrm{~mm}$ hyperechoic linear structures were seen at the corners of the lesion. A digital mammogram was done where four ribbon shaped ultraclips were seen superior, inferior, medial and lateral to the right breast mass. All the four clips were seen on MLO (Medio lateral oblique) view and two clips were seen on CC (Craniocaudal) view. The procedure was uneventful and patient tolerated the procedure well.

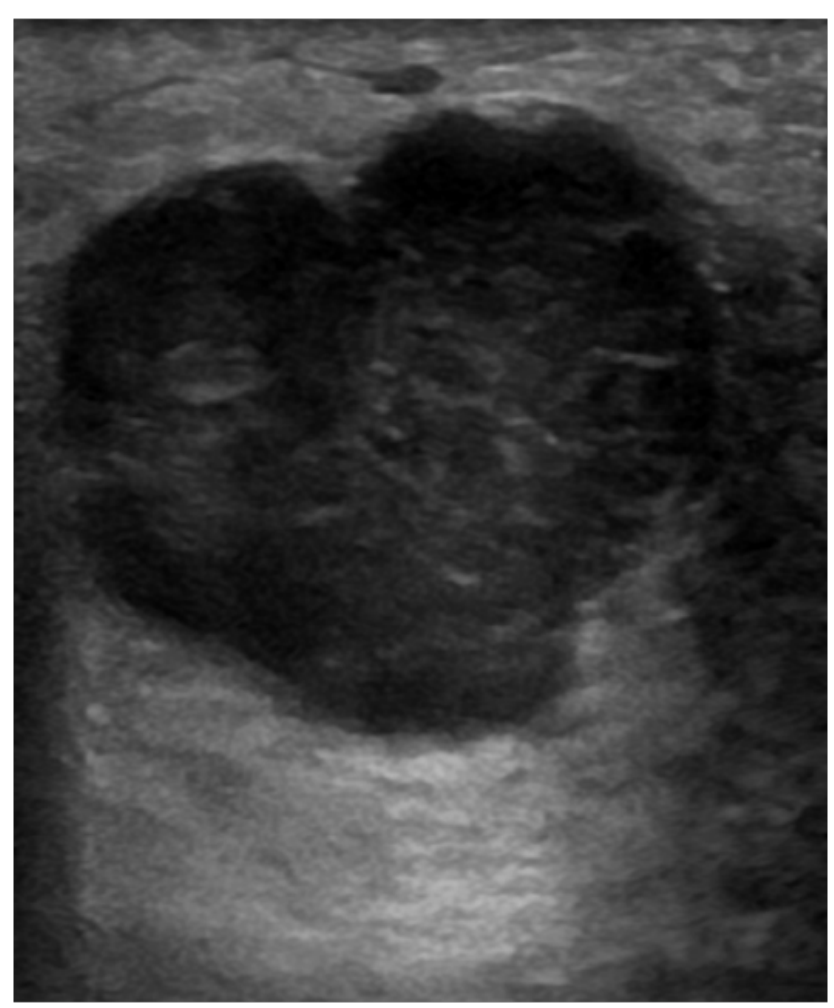

Figure I Ultrasound image of right breast showing circumscribed, irregular hypoechoic mass, measuring $4 \times 3.2 \times 2.4 \mathrm{~cm}$, at 10 O' clock position, approximately $6 \mathrm{~cm}$ away from nipple and $2 \mathrm{~cm}$ deep to skin. The lesion is parallel in orientation and showing no posterior acoustic enhancement or shadowing.

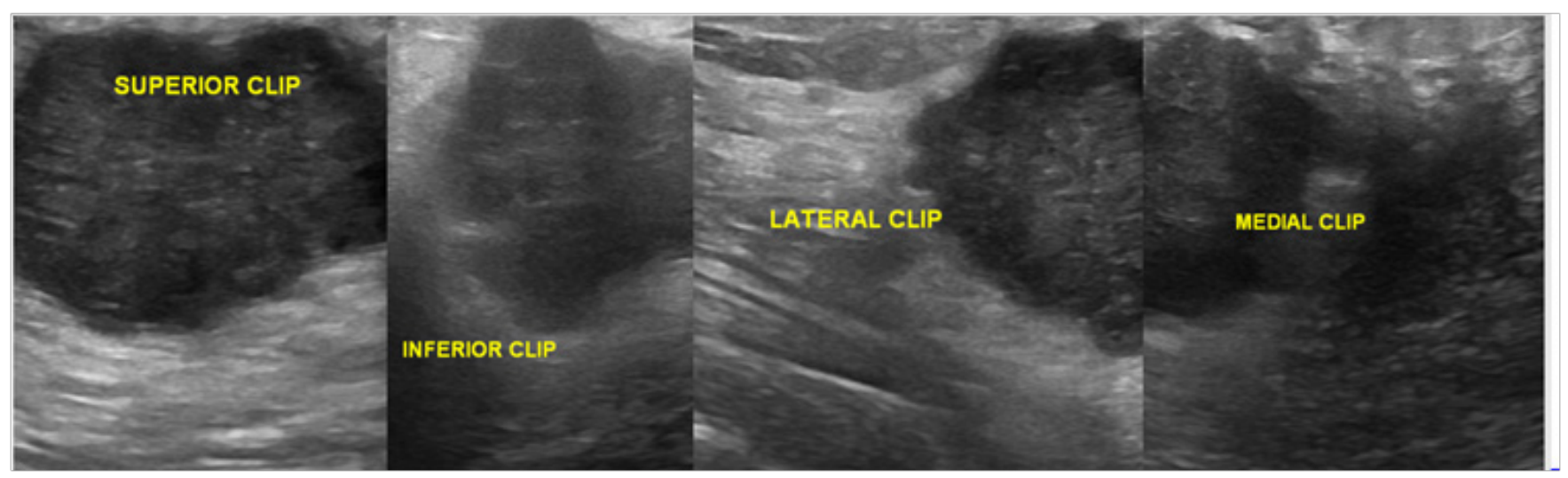

Figure 2 Ultrasound image of right breast showing echogenic clips at superior, inferior, lateral and medial borders of mass. 


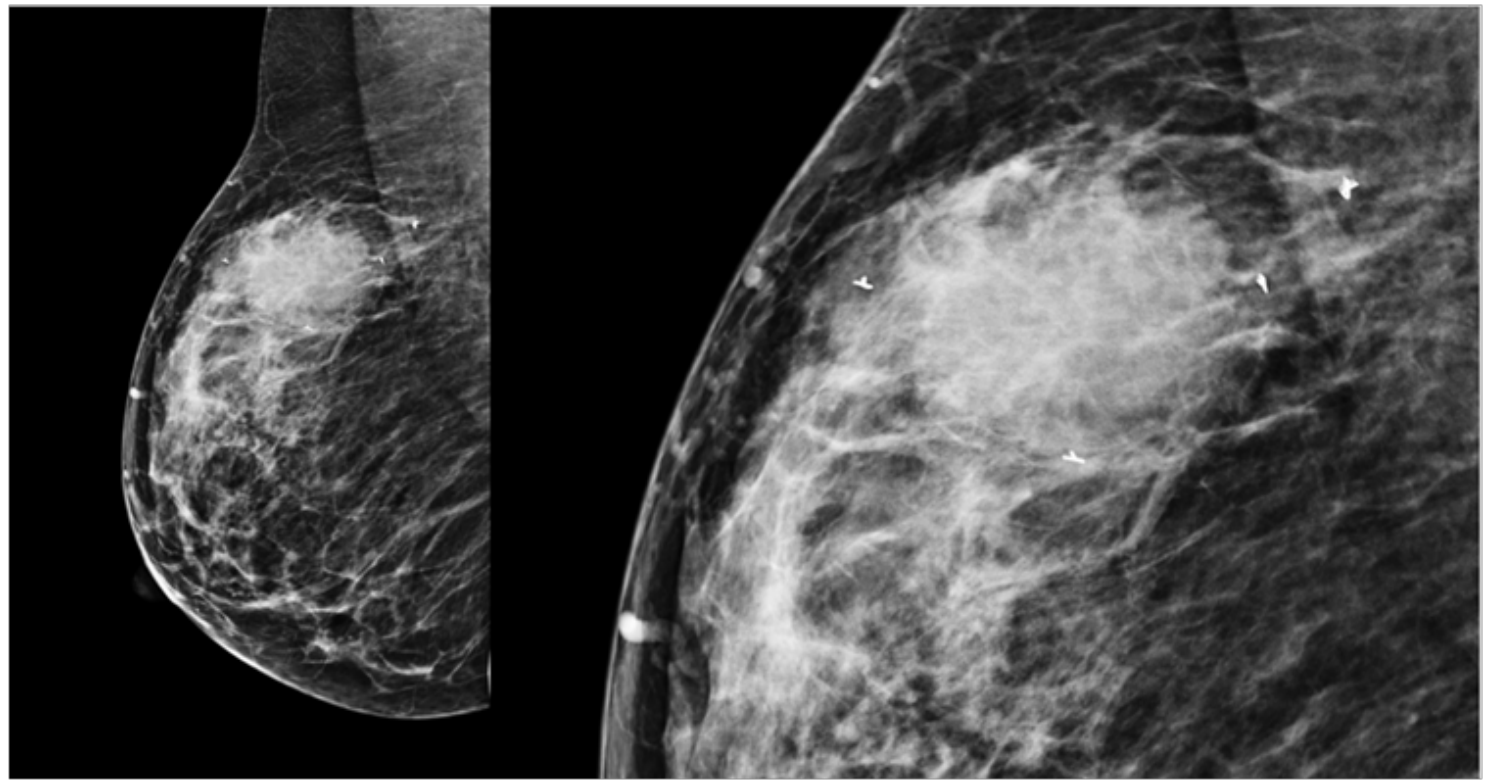

Figure 3 Medio-lateral oblique (MLO) View showing all four breast markers at superior, inferior, lateral and medial borders of mass.

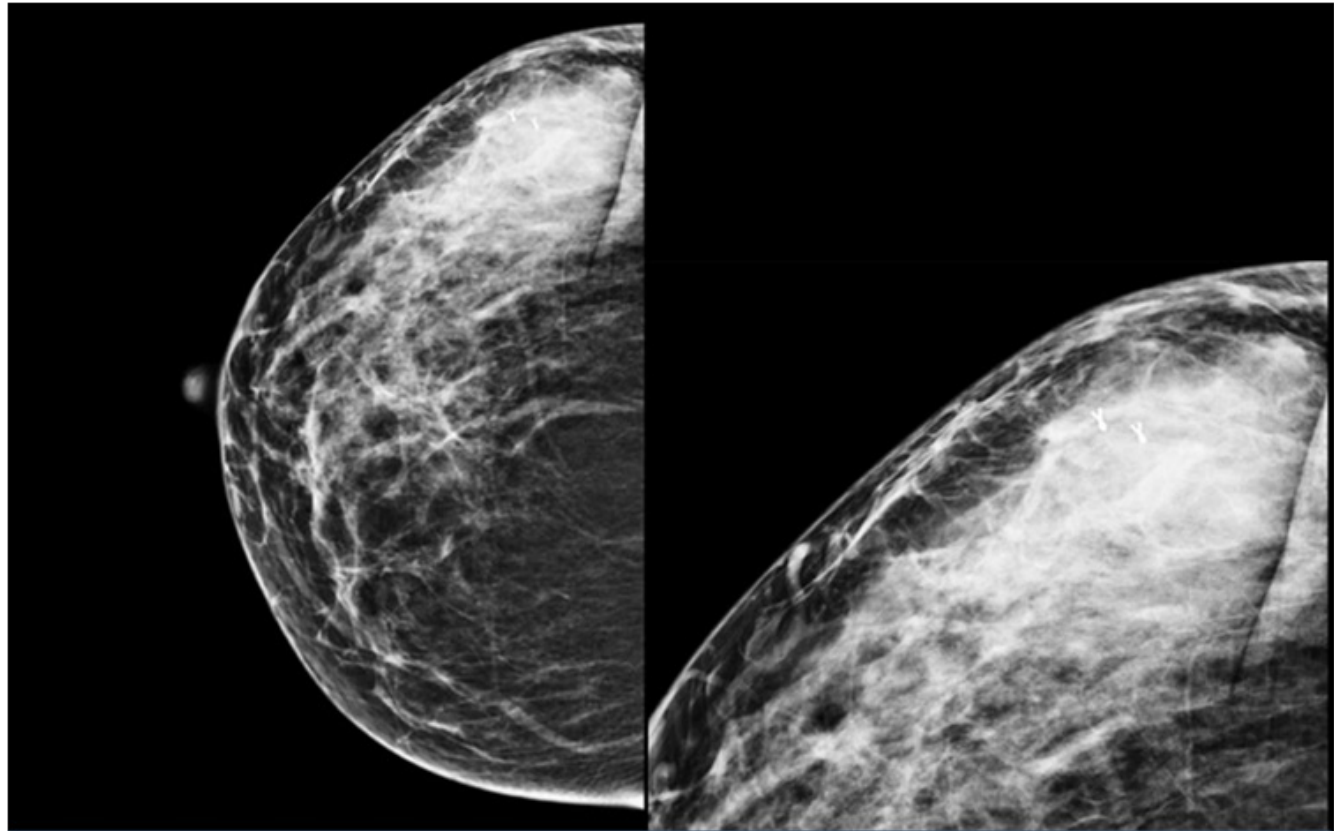

Figure 4 Only two breast markers can be seen on cranio-caudal (CC) view.

\section{Discussion}

Breast tissue biopsy markers are a step towards precision medicine. They help us accurately identify the lesion after a biopsy or post neoadjuvant chemotherapy which may otherwise become very ill defined. They also help us deal with multiple lesions. With availability of ultrasound enhanced breast biopsy markers as opposed to just mammogram visible markers, correlation on different imaging modalities is also possible. Follow up of the lesion becomes easy with breast tissue marker. It also helps in preoperative needle localization thus making the lesion easily accessible on surgery. It marks the margins of extensive disease and guides tumor resection intraoperatively. Pathologist also benefits by knowing the margins of the lesion and region of interest in mastectomy specimen. ${ }^{5}$

With the availability of ultrasound enhanced markers, studies have been conducted on their sonographic visualization in phantoms. In one such study, the UltraClip appeared as a hyperechoic structure. It had a mean diameter of approximately $5.5 \mathrm{~mm}$ relating to the metallic clip in $90 \%$ of the cases. In rest of the $10 \%$ cases, it appeared as a 
hyperechoic tubular structure with a maximum diameter of $9.0 \mathrm{~mm}$ relating to the polyvinyl alcohol polymer. ${ }^{3}$

There have also been studies on the comparison of various commercially available ultrasound enhanced markers. In one such study, only four of the six ultrasound enhanced markers showed statistically significant greater visibility than nonsonographically detected breast tissue markers. Marker size and composition were the contributing factors. ${ }^{1}$ In our case, we deployed ultrasound enhanced markers around the lesion. The marker also had capability to be visualized on mammography and MRI. On post procedural imaging, the markers were seen as hyperechoic linear structures on ultrasonography which corresponded to the length of deployed ultraclips. However, their visualization including their ribbon shape and size were very well visualized on mammography examination. We were surer of the success of the procedure after reading the mammogram. These breast tissue markers hold a promising value in breast intervention and treatment. They are an indispensable tool for better localization and follow up of the lesions. Moreover, they are useful for assessing treatment response after chemotherapy as well. With availability of newer and better visualized markers, their scope is expected to grow in breast intervention.

\section{Acknowledgments}

None.

\section{Conflicts of interest}

The author declares there is no conflicts of interest.

\section{References}

1. Seow J, Phillips M, Taylor D. Sonographic visibility of breast tissue markers: a tissue phantom comparison study. Australas J Ultrasound Med. 2012;15(4):149-157.

2. Margolin F, Jacobs R, Denny S, et al. Clip placement after sonographically guided percutaneous breast biopsy. Breast J. 2003;9(3):226-230.

3. Sakamoto N, Ogawa Y, Tsunoda Y, et al. Evaluation of the sonographic visibility and sonographic appearance of the breast biopsy marker (UltraClip () placed in phantoms and patients. Breast Cancer. 2016;24(4):585-592.

4. https://www.acr.org/Clinical-Resources/Reporting-and-Data-Systems/BiRads

5. Thomassin-Naggara I, Lalonde L, David J, et al. A plea for the biopsy marker: how, why and why not clipping after breast biopsy? Breast Cancer Res Treat. 2011;132(3):881-893. 